OBSERVATIONS ON THE NATURE, ORIGIN, AND TREATMENT OF PUERPERAL FEVER.

By Edward Blackmore, M.D., Edin.

(Continued from page 178.)

CASEs OP puerperal FEVER.

Case I.

A woman, aged 26, subject for years to pain in the stomach and flatulency, requiring pressure for its relief, was delivered of a fine clild on October 18, 1831 , and seized in about twenty-four hours afterwards with pain in the hypogastric region and vomiting. She was seen by her accoucheur twelve hours after the attack, i.e., on the morning of the 20 th, who thought her affeeted with peritoneal metritis.

Bleeding to sixteen ounces; repeated in twelve hours to the same quantity; a blister; nitre with digitalis.

21st. Thirty-six hours from the attack; much complaint of pain over the abdomen and tenesmus; vomiting continuing. Bleeding repeated; a sinapism, and the former medicines.

In the course of the next day the symptoms were mitigated, but the vital powers prostrate.

23rd. The fifth day of illness she was seen by me. The countenance sunken; linbs cold; pulse 130 ; restlessness, sighing, acute pain in the epigastrium; abdomen tumid and tender; tongue clean, but dry; intense thirst and vomiting; the bowels had only been gently opened by a clyster. Her state was manifestly hopeless; effusion into the peritoneum bad begun. A turpentine clyster and a sinapism.

At night there was more distension of the belly, restlessness, vomiting, constipation, (although two clysters bad been given,) and delirium ; and the pulse had ceased. Death in fire hours more:

Inspection.-In the abdomen two pounds of puriform serum, containing albuminous flakes. The parietal peritoneum at the iliac region, (the chief seat of pain,) and at the hypogastrium, very vascular; the uterus healthy; the stomach distended with air and fluids, its texture very lacerable, and at the cardisc end very livid, from extreme vascularity in the peritoneal and muscular coats; the mucous coat healthy. Of the small intestines also, the peritoneal coat was vascular; the mucous natural.

Here the stomach was the seat of the more intense inflammation, the foundation of which had been laid in the previous dyspepsia. It was the second of the fatal cases, out of eight similar, in the course of a fortnight.

\section{Case 11.}

A patient, delivered of her first child by the same gentleman, October 24th, had had slight pains in the bypogastric region, and "water pangs" of the stomach for five months previously. These symptoms were referrible to hard labour in bonnet-ironing, poverty, and grief. On the day after delivety, she was seized with pyrexia, romiting, und severe pain at the lower part of the hypogastrium. These symptoms persisted in the neẍt three duys, in the course of which she was bled largely two or three times; leeched; and blistered ; and had calomel with opium, and nitre with digitalis, in the usual way; and the bowels were freely purged. The blood was much buffed and cupped.

The symptoms persisted to the 29th, the fifth day of the disease, when I saw her. Copious vomiting of a greenish fluid; the hypogastrium tender; tongue loaded; pulse 140, very soft; skin cool and moist; a look of languor and the pupils dilated; the belly not distended; some milk in the breasts. Small doses of turpentine, in gruel, and of calomel with opium, every two hours.

30th. Complains of a dreadful burning pain in the gullet and stomach, with intense thirst, and copious vomiting of a brownish fluid; respiration panting; pulse gone; belly tumid. Death in a few hours on the sixth day of the disease. The third fatal case of fourteen, which had occurred by this time, some of which were afterwards also fatal.

Inspection twenty-four hours, p.m.-In the abdomen a pound and a half of thick turbid puriform serum, with flakes of coagulable lymph, involving particles of pus. The peritoneum of the muscles at the hypogastrium, florid and extremely vascular. The ileum much distended with air; its substance thickened; its serous tunic very vascular, and of a mahogany colour; the mucous coat natural. The uterus contained ragged and dark relics of the placenta ; its substance beneath them appeared natural; the ovaries were much disorganised, softened, and gangrenous; the liver soft, and of an. unhealthy ochry colour; the spleen soft and livid; the stomach exhibited a small star-like spot, of florid vascularity, (explanatory of the symptoms the day before: death.)

\section{CASE III.}

This patient, aged 26, was delirered safely with instruments on October 25th, and continued in the usual state until November 2 nd, the eighth day'after delivery, when she was attacked with griping pains in. the bowels, and a diarrhœa, for which calomel, antimony, and opium, were given with little relief. The purging was stopped on the second day, by chalk and opium; and in a few hours, on the morning of the 6 th of November, she became affected with severe pain and tenderness at the hypogastrium, without previous rigor, and vomiting. The accoucheur saw her in high pyrexia; the pulse very rapid; the secretion of milk suspended; the lochia natural. Bleeding to a pound; eight leeches; a blister; nitre and digitalis.

I saw her on the night of the $7 \mathrm{th}$, forty hours after the acute attuck. The pulse 140 ; respiration very quick; countenance anxious; belly tumid; acute pain of the left lumbar abdominal region, severely aggravated on breathing deeper than ordinary; cose tiveness for fifty bours, although she had taken two ounces of castor oil and purgative pills, and bad four turpentine clysters. Calomel ten grains, opium one grain, and a hot cataplasm. Death in eight houra.

Other fatal cases had occurred to the same practitioner in the interval of the delivery and death of this patient. He lost, certainly, eight out of eighteen cases in little more than four weeks, and afterwards saved. other similar cases by early and judicious bleedling. 
and mercury, at a time when fatal cases were occurring to other accoucheurs.

CASES OF ABDOMINAL INFLAMMATION, ABOUT THE SAME PERIOD, IN THE PREGNANT AND THE PUERPERAL STATE, AND AFTER MISCARRIAGEs.

\section{CASE IV.}

A patient, aged 36 , was delivered by a midwife after a severe but safe labour, at the end of May, 1831, and remained perfectly well, but with slight costiveness, for nearly a fortnight. She was then attacked on the thirteenth day after delivery, with severe pains at the belly and loins, and the lochia were suspended. The next day, Jan. 12th, she was seen by a practitioner, who thought her affected with peritonitis and metritis.

Bleeding to twenty-four ounces; leeches; a blister; calomel five grains, with acetate of morphia, half a grain ; salines, senna, and castor oil.

On the third day, in the morning, she was better; but at noon had a severe return of the former pain, with sickishness; the bowels still costive. In the evening, on the oil acting, she felt better; but in a few hours became again worse, having also severe pains in the head, with intolerance of light and sounds; she was bled at once to twenty-four ounces; the blood cupped and buffed. I saw her twelve hours after this paroxysm, June 14th, the belly tumid, but less painful ; thirst intense; suppression of lochia continuing ; pulse soft, and not quick.

Salines, with colchicum and digitalis; a turpentine clyster every four hours ; calomel with scammony ; a frot linseed poultice on the belly.

In a few hours more the bowels were purged, and the lochia returned.

15 th. The fifth day of the illness; the head relieved; the belly subsided, and only slightly painful on coughing. Sedatires and salines continued.

In the next fortnight a farourable convalescence.

Summary of the treatment:-General bleedings to three pounds ; twelve leeches; two blisters. Of purgatives, castor oil two ounces, scammony 20 grains, cathartic mixture a pint, and several turpentine clysters. Of sedatives, tincture of foxglove four drams, wine of colchicum eight drams; saline solution 40 ounces; calomel half a dram.

$$
\text { CASE V. }
$$

The mother of several children, aged 36 , had felt ill for some weeks, and miscarried in the fifth month of pregnancy, at the beginning of October 1831. A fortnight afterwards, the uterine hæmorrhage returned, and it was followed on the 19th by severe pain in the uterus, and a continued bloody serous discharge, romiting, and fever.

Bleeding to sixteen ounces; ten leeches; a clyster; blue pill and salines, with digitalis and colchicum.

The next day, the symptoms were abated, but the uterine pain, with a quick pulse and a furred tongue, remained for several days. She then took the superacetate of lead, with opium, and had a favourable recovery.

A patient had a safe delivery some months before the rise of the puerperal ferer, in the winter season, and after being costive for a week, and living on game and porter, was attacked with acute pain in the bowels, and beneath the left bypochondrium, followed by vomiting and a severe mucous purging, to twelve stools a day; the milk and lochia were instantly suppressed. Her attendant (now deceased) regarded it as a "bilious and intestinal attack," and gave opium liberally. In a few days all the symptoms of intense enteric-peritonitis were develoyed, and death shortly afterwards ensued.

$$
\text { CAsE viI. }
$$

A patient, aged 32 , was affected in the winter of 1830 , for seven weeks before her confinement, with occasional transient pain in the bowels, (after a fright,) which was usually more severe towards night, and led to an alrine dejection. She had remarked that ber urine was latterly high-coloured, and that animal food disagreed. Hydrargyrum cum creta and acetate of morphia were given with relief on various occasions. On December 13th, she eat beef at dinner, and at night had more pain, which again increased the next morning, with more shiverings, and the bowels moved twice. After taking the above-named medicines she seemed better; but at one p.m., violent vomiting occurred, on taking some gruel, with agonising pain at the left side of the abdomen, and an irritating disposition to a stool; to this speedily succeeded thirst, whiteness of tongue, and diffuse abdominal tenderness, without uterixe pains. She was supposed to be within two weeks of the full period of pregnancy. At three p.m., blood was rapidiy drawn to eighteen ounces, with marked relief, without syncope ; the rigors, which had returned sererely before the rein was opened, ceased instantly after the operation. The blood was buffed and cupped. Four hours afterwards there was again a return of abdominal tenderness, with crampish pain in the uterus. The next morning the pulse was 110 and strong, and the thirst severe. Some castor oil and calomel had procured good stools. In the course of the day the pulse was reduced by colchicum and salines; the ensuing night was quietly passed, and on the following morning natural uterine pains came on, which in a few hours terminated in an easy parturition.

If the contagion of puerperal fever, and the atmospheric conditions which conduce to its operation, had then existed, there can be little doubt that this case would have exhibited all the phenomena of the more sthenic form of the specific disease.

$$
\text { CASE viII. }
$$

A woman, aged 30, at the end of March, 1831, had an abortion, with much hæmorrhage; followed in six weeks by a return of flooding, with rigors, and pain in the belly, more so at the iliac and lumbar regions. She was seen a day or two after the attack, on May 15th, by her accoucheur, who bled her largely, and repeated it the next day. On the $17 \mathrm{th}$, a blister was applied, and the usual saline medicine given. In the next eight days the symptoms were slightly relieved, but she was confined to her room. On the 26th, a severe return of pain in the left side of the abdomen; with nausea and retching. The next day, an exacerbation of pain in the right side of the belly, with vomiting, and costivenean. 
Leecbes used; purgatives neglected. The 28th bled in the morning to trenty-four ounces. Blood not inaned.

I saw her at night, on the fifteenth day of illness, sufering under pain in the right hypochondrium, abdomen, and loins, 80 severe as to impede breathing and lying-down; pressure intolerable; the pain in serere paroxysms, with occasional voniting; pulse 140 ; respiration quick; bowels costive for three days; urine high-coloured; little heat of skin; tongue paded at the root. Calomel, with colchicum and acetate of morphia erery three hours; salines, with digitalis; an emollient poultice, to be followed by a blister, and clysters.

The next day, the fourth of the severe relapse, great relief of pain; pulse 100; the bowels imperfectly eracuated. Castor oil, scammony, and blue pill.

30th. An improvement; but pain beneath the scapula, impeding respiration; still inefficiently purged. Calomel, scammony, and rhubarb. In the evening, some bloody fibrinous discharge from the vagina.

3lst. Better; salines, with colchicum.

Is the next four days the pulse quick, the other symptoms better. Salines, with digitalis; stmall doses of acetate of morphia, with blue pill.

June 4 th. After dining on fish, and having been costive, attacked with pain in the bowels and head, delirium, and feverishness. On taking calomel and rhubarb, she was purged of fetid slimy stools. The nezt day, intestinal pains, obliging her to compress the belly; tongue white and slimy. Castor oil and a clyster. In a few hours, faintness, cold sweats, abdominal tenderness; the bowels not having acted. Twelve leeches; a clyster, and castor oil, with liquor potassæ.

The next day, 24th from the attack, paroxysms of spasmodic pains in the bowels, which were still imperfectly evacuated; a white tongue, and quick pulse. Clysters ; a sinapisn ; scammony, ipecacuanha, and blue pill, with acetate of morphia.

7th. More abdominal tenderness; leeches, and the former medicines. The next day, purged of many biliows stools with great relief; efferrescing salines, and blue pill continued.

9th. Restlessness in the night, from a storm; sleep after an opiate; feelings improved after free purging of bilious stools. Laxatives ; mercury omitted; a fannel roller on the belly. By persisting in the use of laxatives during another week, her corvalescence was confirmed after a month's illness.

Summary of treatment.-Bleeding to fifty-six ounces; leeches; mercury, acetate of morphia, colchicum, digitalis ; salines, purgative nedicines, and clysters ; blisters and poultices.

Her case, on my first seeing her, appeared dangerous, and I think would have been fatal, if it had been regarded either as one of pure inflammation to be treated then by further bleeding, or as enterodynia, requiring chiefly opiates. The state of the pulse and tongue clearly indicrted the use of morphia with inercury, and the bowels urgently required purgatires. If puerperal ferer had then prevailed, the prognosis wöuld have been despondent.

\section{Case 1x.}

A woman, aged 39, had an easy parturition, August 29th, 1829, with little hæmorrhage. The placenta came away instantly, and was probably unhealthy, for the child was puny, and of a dingy yellow hue; the labour premature by nearly four weeks. She had complained of pain in the head and right flank for two months previously, and had a severe cough with mucous serous sputa, and much dejection of spirits.

A few hours after delivery she was affected with rigors, succeeded by fever, vomiting, and a return of pain in the right flank, head, and limbs. Some castor oil had been taken, and a good stool obtained. The symptoms had persisted for sixty-four hours when I saw her; the beadache had then been relieved by epistaxis; the belly was very painful and tumid; the bowels griped and costive; pulse 100, and tense; romiting urgent; yet the expression of countenunce good, and the lochia going on. In a few hours, at the end of the third day from the acute attack, the symptoms were relieved by the remedies, - a large bleeding, leeches, and calomel. During the next dny this mitigation of the symptoms continued; but the pain in the epigastrium returned on taking gruel and medicine; pulse 120, tongue white and coated; bowels well purged.

On the fifth day after delivery, one hundred hours from the attack, she had again a severe rigor, followed by profuse sweating; but when seen by me shortly afterwards, the pulse was 80 , and soft. She remained better through the sixth day, but the lochia had ceased to flow, and a slight cough returned; pulse 90 , soft.

The following morning, mild delirium, with fever; the countenance vacant and dejected; yet she expressed herself relieved. The eight day, a slight fetid bilious purging ; the pulse more rapid; skin icteritious; mouth sore from mercury; yet she made little complaint. Towards night, the pulse and breathing became rery quick, tremors ensued, the whole state like the last stage of a putrid fever. The next day, prostration greater; pulse 130. On the 10th day, after the cordials, a deceitful amendment, the lochia reappeared after having ceased four days. She sunk the following day.

The treatment consisted of a bleeding to twenty ounces, on the third day; six leeches; calomel, in large doses; sedatives, purgatives, turpentine clysters, blisters, and at the end, cordials in a large quantity.

Inspection thirty-two hours, p.m.-The skin, and subcutaneous fat, of a deep dark yellow colour. On the exterior of the right lung a dark corrugated indurated spot, like a gangrenous eschar, beneath it a large cavity, containing fetid pus; its boundaries gangrenous; two smaller similar cavities in the left lung; the larger bronchial tubes dark, vascular, containing mucous; the heart soft, holding dark serous blood; uir in the pleura and pericardium; the latter membrane very rascular, bolding three ounces of serum. The blood in the body generally fluid, dark, and serous; the abdomen sunken; the right ovary entirely disorganised and gangrenous, containing some fetid pus, adberent to the uterus; the left, in its colour and 
texture, was like tanned leather; the uterus black and softened to a half of its thickness, where the ovary adbered, and at its neck. In its interior, at the fundus, was a gangrenous mass, like the relics of the placenta; the whole of the mucous cout pulpy and black. The stomach, in its mucous cont many ulcers with a livid base; the other portions pulpy, dark, and exhaling a fetid odour. The intestines and peritoneum natural ! Much dark sandy bile in the gall-bladder.

This remarkable case much resembles, in its symptoms, the usual description given of the more malignant variety of puerperal fever. I believe, however, its origin and nature to be different, and the course of its symptoms certainly was 80 , from that of the specific ferer.

\section{NEW OPTICAL INSTRUMENT TO FACILITATE THE EMPLOYMENT OF THE SPECULUM UTERI.}

TO THE KDITOR OF THE PROVINCIAL MEDICAL AND -

Sir, gURGICAL jOURNAL.

Having frequently felt the want of an optical instrument, which would accurately show the state of the parts, in examinations of the os and cervix uteri, by means of the speculum, the plan of looking through a reflector suggested itself to me, and on this principle I had an instrument made by Mesers. Abrahan and Dancer, of Manchester, which I have used for some time past, and have found to answer the purpose exceedingly well, It is also well adapted for examining the ear or throat, and can be used in either hand as may be needed.

Fig. 1.

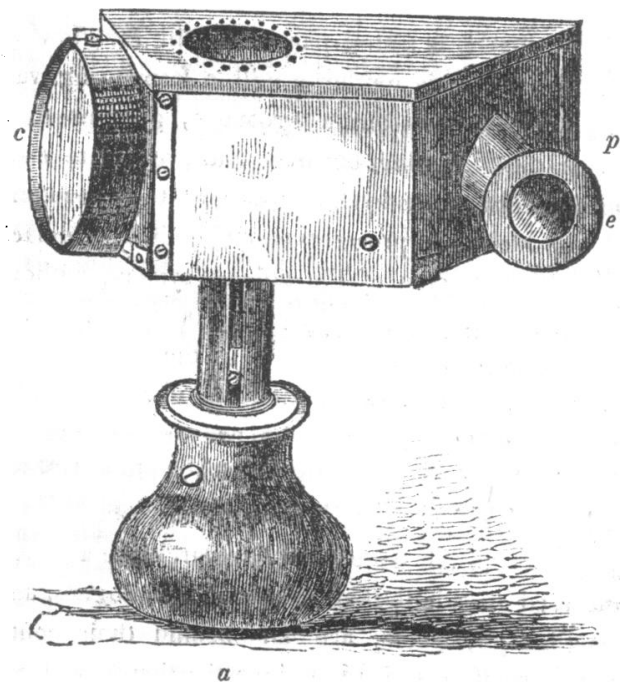

Figure 1 is a drawing of this instrument. It consists of a hollow body, bevelled off at one end, and perforated at the top and bottom. The handle $(a)$ fits into the lower perforation, and is so constructed as to hold a wax taper, which may be elevated and depressed by means of a screw, 80 as to adjust it to the focus of the internal concave mirror (c.) When a lighted taper, thus adjusted, is introduced into the body of the instrument, the rays of light falling on the mirror (c), are thence reflected towards the opposite or bevelled end $(p)$, which is lined with a plane mirror, so placed as to again reflect the rays of light, and to throw thein directly, through the tube of the speculum, to which the instrument is connected, upon the parts to be examined. The bevelled end of the instrument, together with the plane mirror, is perforated, and an eye-piece $(e)$ inserted, which is thus continuous with the speculum, and enables the observer to see the parts upon which the light is thrown. The eje-piece has a loose cap, into which may be fitted any lens or lenses, of various focal lengths, as may be required.

Fig 2.

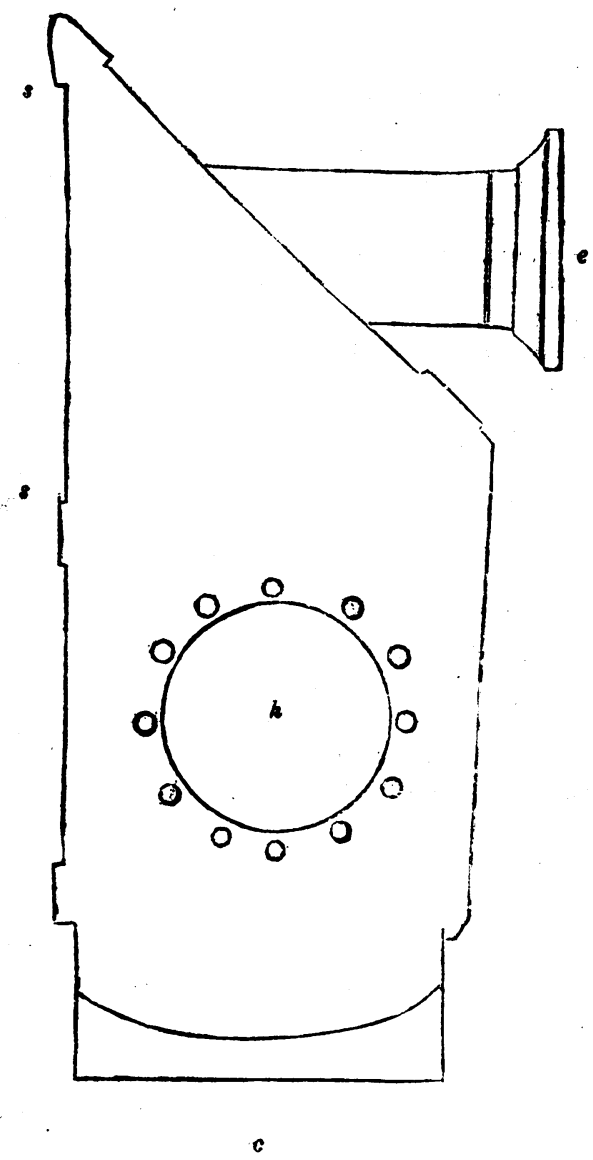

Figure 2 is a diagram of the instrument:-c. The concave mirror.

e. The eye-piece inserted into the bevelled end or the instrument, and perforating the plane reflector.

$h$. The perforation through the body of the instrument, where the handle fitted with the wax-tapers, is introduced.

8. . . 8. The part by which the instrument is connected with the speculum.

I remain, Sir, your obedient servant, J. HUTCHINSON, M.R.C.S.

Cheetham Hill, Manchester, Feb. 6, 1845. 